\section{Simple method of aligning cylindrical air-spaced elements}

\author{
John P. Lehan, a MEMBER SPIE, Theodore Hadjimichael,, \\ and David A. Content, ${ }^{b}$ MEMBER SPIE \\ ${ }^{\mathrm{a}}$ Center for Research and Exploration in Space Science \\ and Technology, NASA Goddard Space Flight \\ Center and Department of Physics, University of Maryland, \\ Baltimore County, 1000 Hilltop Circle, Baltimore, \\ Maryland 21250 \\ ${ }^{\mathrm{b}}$ NASA Goddard Space Flight Center, Greenbelt, \\ Maryland 20771 \\ 'Swales Aerospace, 5050 Powder Mill Road, Beltsville, \\ Maryland 20705 \\ E-mail: Theodore.J.Hadjimichael.1@gsfc.nasa.gov
}

\begin{abstract}
A simple method is found to align multielement cylindrical lenses. The method employs only equipment found in most optical shops: a precision flat mirror and a Fizeau interferometer. A combination of narrow reflection interferograms from the lens surfaces, combined with cat-eye double-pass interferograms, is employed to align the elements. (0) 2007 Society of Photo-Optical Instrumentation Engineers. [DOI: 10.1117/1.2750656]
\end{abstract}

Subject terms: anamorphic optical systems; cylindrical optics; optical alignment.

Paper 070171LR received Feb. 23, 2007; revised manuscript received Apr. 10, 2007; accepted for publication Apr. 20, 2007; published online Jul. 11, 2007.

\section{Introduction}

Using systems of cylindrical elements for lens design has been proposed for many years because of their unique properties, chief among them their anamorphotic (or anamorphic) properties. Slyusarev ${ }^{1}$ gives the most thorough discussion of the aberrations of such systems. Most of the early systems built, however, did not live up to their potential primarily due to the low quality of the components. Starting in the 1980's, lens fabricators have improved the quality of cylindrical elements to the point that the realization of multiple-element systems with good performance is now feasible. In addition, methods of testing cylindrical surfaces are now approaching maturity. The most common for surface testing today is normally incident null testing employing a diffractive optical element (DOE). ${ }^{2}$ This approach limits the size (DOE fabrication method) and $F$ number (minimum feature size) testable. With the advent of better subaperture stitching techniques brought about by computer computational power, however, the size constraint is becoming less problematic. A good second method employs two DOEs with the part viewed in glancing incidence. ${ }^{3}$ This method has the distinct advantages of not being limited in $F$ number and the possibility of testing both surfaces at once, but the disadvantages of the height resolution being a function of the minimum feature size and an indistinct pupil, making fiducialization (and thus error correction) difficult. The literature on the methodology to

0091-3286/2007/\$25.00 @ 2007 SPIE assemble and test such multielement cylindrical lenses, however, is sparse. We attempt to fill this void to some extent. We first touch on the measurement of singlets (those properties needed for assembly), then the alignment of multiple element systems.

\section{Preliminaries}

Table 1 summarizes the metrology required to optically characterize a cylindrical lens. (Note that this table is shorter than the corresponding one in Ref. 3 , because we are not interested in the surfaces that do not participate in the formation of images but are rather used for mechanical reference.) Here we refer to a cylindrical lens as a lens with two toroidal surfaces, the radii of curvature in one direction, sufficiently large so that it can be measured by comparing it to a flat, and that direction is nominally parallel on $S 1$ and $S 2$. Note that this pragmatic definition varies with the axial extent of the lens, with smaller lenses allowing shorter radii of curvature.

When dealing with cylinders as defined, the irregularity quoted by most manufacturers is the combination of the axial radius and irregularity of Table 1 . We split these because there are instances where some axial power can be used to advantage in anamorphotic system design. Thus, in Table 1, irregularity is reserved for terms beyond the second order. We also note that there is no azimuthal wedge specified. This is because it is optically equivalent to axes displacement, as illustrated in Fig. 1.

Quantities in italics in Table 1 are not addressed in this work, so the reader is referred to Ref. 2 and especially Ref. 3 for methods of obtaining these quantities. Note that for true cylinders (axial radius of curvature infinite), the displacement of the axes between surfaces is only perpendicular to the axes (azimuthally or parallel to A-B in Fig. 1); however, in our pragmatic definition, axial displacement of the toroidal surface vertices should, in general, be measured as well and is included via the axial prism term.

\section{Singlet Characterization prior to Alignment}

This process is reasonably straightforward. It is assumed that the surfaces ( $S 1$ and $S 2)$ have been characterized previously, as described in Refs. 2 or 3. The first step is to place the lens in front of the Fizeau interferometer (we employed a Zygo VeriFire ${ }^{\mathrm{TM}}$ for this task because the variable intensity feature made looking at the antireflectioncoated surfaces easier). The lens is aligned axially so that the narrow vertex (front and rear) interferograms appear

Table 1 Summary of cylindrical singlet metrology desired for precision cylindrical lens assemblies. (See text for discussion.) Axes displacement is usually called decenter by manufacturers in analog-tospherical optics. Axial radius, axial clocking, and axial prism are not normally specified by cylinder fabricators.

\begin{tabular}{lcccc} 
Surface & Axial radius & Irregularity & $\begin{array}{c}\text { Azimuthal } \\
\text { radius }\end{array}$ \\
Glass & Index & Homogeneity & & \\
Element & $\begin{array}{c}\text { Axes } \\
\text { displacement }\end{array}$ & Axial clocking & $\begin{array}{c}\text { Axial } \\
\text { prism }\end{array}$ & Thickness \\
\hline
\end{tabular}




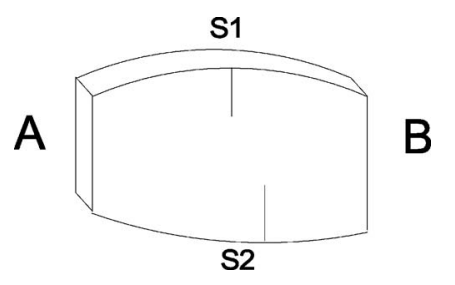

Fig. 1 Top view of a cylindrical singlet illustrating how a displacement of the axes of the cylindrical surfaces results in an azimuthal wedge (wedge in the A-B direction). Note that this same lens would result from tilting $S 2$ relative to $S 1$. The vertices of the surfaces are marked with small lines.

[Fig. 2(a)]. By rotating the lens about an axis (roughly) through the nominal azimuthal center of curvature of $S 1$, one can determine which is the front and rear surface. Displace the two interferograms slightly, and take the data. (To aid in the taking of the interferograms with phase-stepping interferometers, some small defocus can be introduced without deleterious effects. ${ }^{4}$ ) From this measurement, we can obtain both the axial curvatures and axial wedge. If desired, the lens can be remeasured with $S 2$ facing the interferometer, providing a more accurate determination of the $S 2$ axial curvature (since $S 1$ and the refractive index of the lens need not be taken into account.)

Next, we focus the interferograms and overlap on the center line of the lens. Measure the displacement of the center lines of the interferograms at the top and bottom of the lens. This is a measure of the axial clocking of the surfaces [see Fig. 2(b)]. (Alternatively, image analysis allows one to determine the RMS slopes if there is greater irregularity of the narrow interferograms.) The precision of the determination of the axial clocking step is typically determined by the pixel size of the camera in the interferometer. Of note is that very parallel axes will brighten appreciably when coaligned to the interferometer (assuming laser irradiation) along their entire length, but only near the midpoint of the lens if clocking is appreciable.

With the $S 1$ and $S 2$ vertex interferograms overlapping [at the midplane axially-see Fig. 2(b)], position a flat so that the best cat-eye interferogram is obtained (for a positive lens). Remove the lens to determine the wedge of the transmitted beam. This allows an independent measure of the axial wedge (typically better than from the line returns).

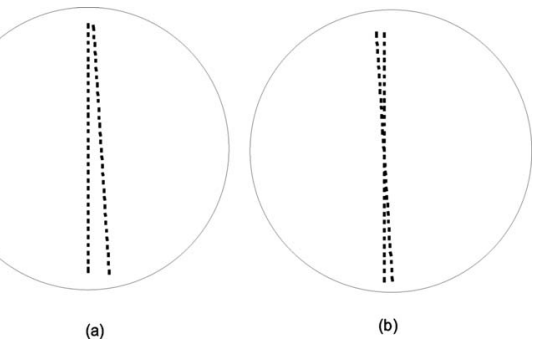

Fig. 2 Drawing of the two line interferograms from the cylindrical surface vertices for a singlet: (a) for axial wedge and axial curvature determination, prior to overlapping, and (b) overlapped for axes clocking determination. (Note: the clocking of axes is greatly exaggerated for illustration purposes.)
(Note, if a negative lens is being measured, overlap all the line interferograms, the two original plus the double-pass transmitted one.)

\section{Air-Spaced Multiplet Assembly}

The procedure described here is for a focusing lens system operating at infinite conjugates, but could be readily adapted to other conjugates. The alignment of systems with virtual images would require the addition of another element during the alignment to make the combined system focusing.

With the singlet information obtained, the designer can determine the best performance expected for the lens assembly. During assembly, six degrees of freedom are needed in general. It proves convenient that we assemble the lens in such an order that the first two singlets placed have the minimum wavefront error in combination. Although this is not an absolute requirement, it does minimize the uncertainty in the placement of the initial pair of elements.

First, a singlet is aligned to the interferometer. This is done as in the singlet measurements before by having both line returns overlapping and minimizing the combined tilt fringes parallel to the cylinder axis (the long radius of curvature direction). The next lens is placed in its approximate location (mechanically). The second lens is tipped and tilted until its vertex interferograms overlap with those of the first. (Some small axial displacement can be introduced to separate the line interferograms temporarily so that the axial tilt of the second lens can be minimized, but it is better to work toward the interferometer so that one can block the returns from the farthest lens.)

Next, a retroreflecting mirror is placed after the lens assembly and a double-pass cat-eye interferogram obtained. This allows further tip adjustments to compensate for axial wedge in the elements along with wedge in the air space between the elements. (Unlike in axisymmetric elements, the cat-eye interferogram has only bilateral symmetry, so the axial aberrations are apparent.)

(Note: Oddly, imperfect alignment in this procedure [as shown in Fig. 2(a)] can result in a tilt in the interferogram in the azimuthal direction, since the optical paths are not exactly matched on the outward and return paths, despite the assumed bilateral symmetry. If this occurs, go back to line interferograms and realign to the interferometer [Fig. 2(b)], and the problem should disappear.)

If the motions in the lens housing are coupled, then one will need to iterate between the line returns and the cat-eye measurements. This is achieved by simply blocking the retroreflecting mirror.

When the first combination of singlets has the minimum axial aberration and proper azimuthal aberration (from modeling of the singlets), the next element can be added and the process repeated. The residual azimuthal aberration is usually the cylindrical equivalent of spherical aberration, hence the desire to start the procedure with the two elements that minimize this quantity in combination.

When the process is completed, removing the aligned lens assembly from between the interferometer and cat-eye mirror will permit us to determine the overall wedge-just as in the singlet case. If this wedge is deleterious to the application, careful notes should be taken on the orientation 
for the various singlets in the assembly, so that the singlets can be placed into the overall assembly in the proper orientation to minimize the final wedge.

\section{Discussion}

We note that, while the previous procedure is sufficient to align a cylindrical lens system, in the sense that all the relative lens positions needed to minimize alignment errors are tracked, it is not adequate to calibrate the performance, since the cat-eye interferograms mix the right and left halves of the system. Also of note is that the focal line of the system must be real and accessible to obtain the cateye.

We have not provided explicit expressions for the theoretical accuracy obtainable by this methodology, because they depend on the details of the design, fabrication quality, and the imaging characteristics of the interferometer employed. Thus, this analysis should be undertaken on a caseby-case basis.

We have applied the prior procedure with success to a moderately fast $(F / 1.5)$ cylindrical null lens for testing the mirror segments for the Constellation-X X-ray spectroscopy telescopes $(\mathrm{SXT}){ }^{5}$ In this case, some axial curvature is desired, because the mirrors themselves have axial curvature by design (a radius of about a kilometer), which improves the telescope imaging performance. ${ }^{6}$ Because we were interested in measuring the net axial curvature as a check on our singlet metrology, our mirror was about 1/50 wave RMS and almost free of second-order error. (More generally, any well-characterized mirror could be used, but a better mirror simplifies the real-time interpretation of the data while aligning.) The system performance after alignment by this procedure was found to be within the uncertainties of the optical model prediction for a perfectly aligned system, which included the imperfections found during the singlet metrology. We have found that the lens performance is limited by the fabrication errors in the individual elements rather than alignment errors (within our ability to measure).

\section{Acknowledgment}

We wish to acknowledge the support of the National Aeronautics and Space Administration through the Constellation-X mission. J. Lehan also wishes to acknowledge the guidance of the Lord in reaching a successful conclusion in this research.

\section{References}

1. G. G. Slyusarev, Aberration and Optical Design Theory, 2nd ed., pp. 571-594, Adam Hilger, Bristol (1984).

2. Zygo Corporation, "Testing cylindrical surfaces with computer generated holograms," Application Note, see http://www.zygo.com/?/ library/appnotes/appnotes.htm.

3. K. Mantel, N. Lindlein, and J. Schwider, "Simultaneous characterization of the quality and orientation of cylindrical lens surfaces," Appl. Opt. 44, 2970-2977 (2005).

4. D. A. Content, D. Colella, C. Fleetwood, T. Hadjimichael, T. T. Saha, G. Wright, and W. W. Zhang, "Optical metrology for the segmented optics on the Constellation-X soft x-ray telescope," Proc. SPIE 5168, 207-218 (2004).

5. J. P. Lehan, T. Hadjimichael, D. A. Content, and W. W. Zhang, "Design and fabrication of refractive nulls for testing the segmented mirrors of the Constellation-X spectroscopy X-ray telescope (SXT)," Proc. SPIE 5900, 59001D (2005).

6. T. T. Saha and W. W. Zhang, "Equal curvature grazing-incidence X-ray telescopes," Appl. Opt. 42, 4599-4605 (2003). 